\title{
Pancytopenia with focal serous atrophy (gelatinous transformation) of the bone marrow
}

\author{
Gregory P. Kaufman • Jeremy T. Larsen • \\ Michelle A. Elliott • Matthew T. Howard
}

Received: 4 May 2014 / Accepted: 9 May 2014 /Published online: 25 May 2014

(C) Springer-Verlag Berlin Heidelberg 2014

Dear Editor,

While the myriad of clonal and non-clonal causes of pancytopenia are well outlined [1], we found the following case particularly illustrative to the practicing clinical hematologist, as it highlights the importance of careful morphological evaluation of the marrow for focal abnormalities in an otherwise complex patient. Briefly, a 72-year-old male was admitted to the ICU with hyperglycemia and metabolic derangements and found to have new pancytopenia and severe neutropenia ( $\mathrm{Hgb}$ $8.9 \mathrm{~g} / \mathrm{dL}$, PLT $140 \times 10^{9} / \mathrm{L}$, WBC $1.2 \times 10^{9} / \mathrm{L}$, ANC $460 / \mu \mathrm{L}$ ). He had a history of stage IV mantle cell lymphoma treated with 6 cycles of bendamustine and rituximab, with the last cycle 5 months prior to index hospitalization, and had obtained marked response. CT scans 2 months prior to admission and again with the current hospitalization showed no evidence of worsening lymphoma. It was noted that the patient had obtained count recovery following chemotherapy with normal $\mathrm{CBC}$ indices documented just 3 months prior to the current hospitalization. His recent clinical history was one of progressive debility and functional decline, unintentional weight loss

G. P. Kaufman · J. T. Larsen • M. A. Elliott

Department of Internal Medicine, Mayo Clinic, Rochester, MN, USA

J. T. Larsen • M. A. Elliott

Division of Hematology, Mayo Clinic, Rochester, MN, USA

M. T. Howard ( $\square)$

Division of Hematopathology, Mayo Clinic, Rochester, MN, USA

e-mail: Howard.Matthew@mayo.edu

M. T. Howard

Department of Pathology, Mayo Clinic, 200 First Street SW, Rochester, MN 55905, USA
(30 lbs over the last 6 months), and he would be accurately described as malnourished and cachectic in appearance. Initial workup for infection, hemolysis, or other causes of cytopenias was unrevealing. A bone marrow biopsy was obtained, and images from the bone marrow core biopsy are shown in Fig. 1. There was no evidence of recurrent lymphoma or other clonal process explaining his new pancytopenia. Rather, patchy areas of hypocellularity and fat cell atrophy were seen in association with a generalized decrease of otherwise normal hematopoiesis and focal deposition of an eosinophilic proteinaceous material.

Serous atrophy, or gelatinous marrow transformation (GMT), is an uncommon finding that while not specific for one particular disease entity, can be seen as a severity indicator of general illness. While the pathogenesis of GMT is not completely understood, it has been associated frequently with weight loss and malnutrition in as high as $70 \%$ of cases [2]. The largest GMT series have reported a strong male gender predominance, and note other frequently observed associated conditions such as carcinomas, lymphomas, chronic infections, clonal myeloid disorders, status post recent chemotherapy, alcoholism, anorexia nervosa, and heart failure [2-4]. Mild normocytic anemia is the most common cytopenia reported with GMT, whereas pancytopenia with severe neutropenia as seen here has been less commonly reported. Interestingly, GMT may have focal or diffuse involvement of the marrow space as our case demonstrates. This has been speculated as a possible reason for its relatively uncommon reported incidence in large series of reviewed marrow biopsies, as a single iliac crest biopsy may not include such focal areas. 


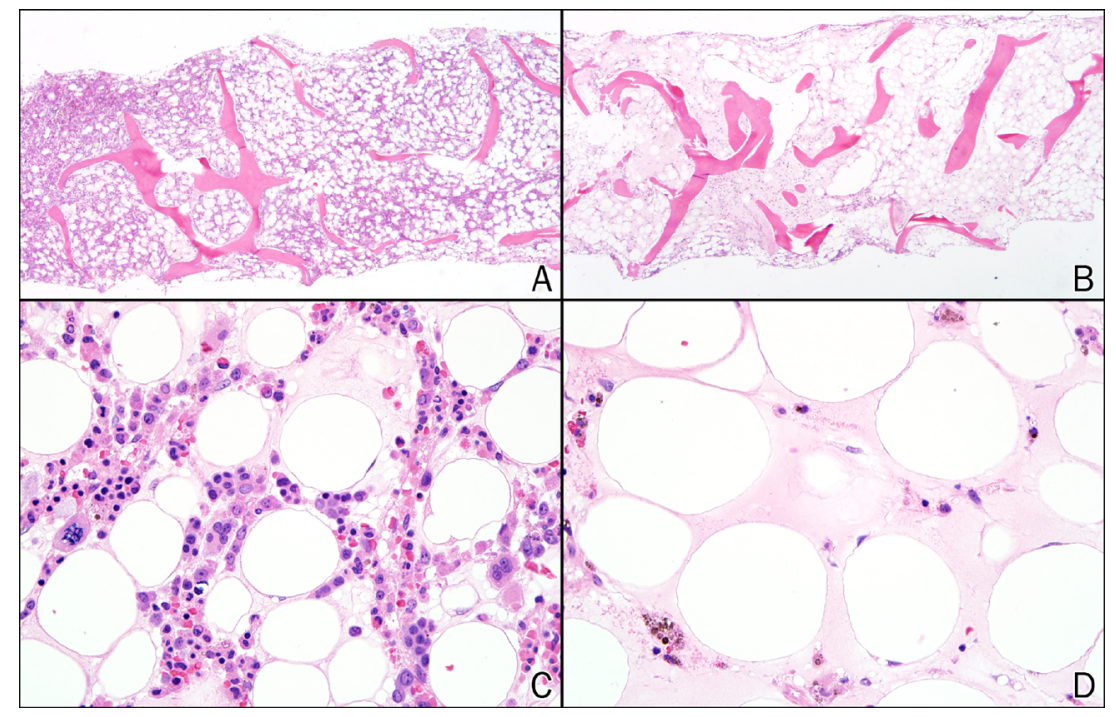

Fig. 1 Bone marrow core specimen demonstrating focal serous atrophy and hypocellularity. A unilateral bone marrow biopsy with two trephine cores was obtained. a One bone marrow core shows relatively normal cellularity for age and normal appearing hematopoiesis. Subtle eosinophilic deposits are noted between areas of hematopoiesis. (hematoxylin and eosin, $\times 20$ magnification). b Another area of the same marrow biopsy showing marked hypocellularity with prominent amorphous smooth to slightly fibrillar eosinophilic protein deposits between areas of reduced hematopoiesis (hematoxylin and eosin, $\times 20$ magnification). c Higher power view of the marrow core shown above in (a). The hematopoiesis appears normal, with atrophic adipocytes surrounded by eosinophilic proteinaceous deposits (hematoxylin and eosin, $\times 400$ magnification). d Another area of the marrow core from above (b) at higher magnification. Hematopoiesis is extremely sparse; scattered hemosiderin laden macrophages and amorphous eosinophilic protein deposits are present (hematoxylin and eosin, $\times 400$ magnification)
This case highlights the potential detrimental effect of severely compromised nutrition on hematopoiesis and the importance of careful morphological review of the bone marrow for abnormalities that can provide insight into the cause of cytopenias in an otherwise complex patient.

Conflict of interest The authors declare that they have no conflict of interest.

Informed consent was obtained from the patient to publish this case.

\section{References}

1. Devitt K, Lunde J, Lewis M (201) New onset pancytopenia in adults: a review of underlying pathologies and their associated clinical and laboratory findings. Leuk Lymphoma. 3;Early Online:1-7.

2. Böhm J (2000) Gelatinous transformation of the bone marrow. Am J Surg Pathol 24(1):56-65

3. Michael P (1930) Gelatinous degeneration of the bone marrow. J Pathol Bacteriol 1930:533-538

4. Seaman J, Kjeldsberb C, Linker A (1978) Gelatinous transformation of the bone marrow. Hum Pathol 9(6):685-692 